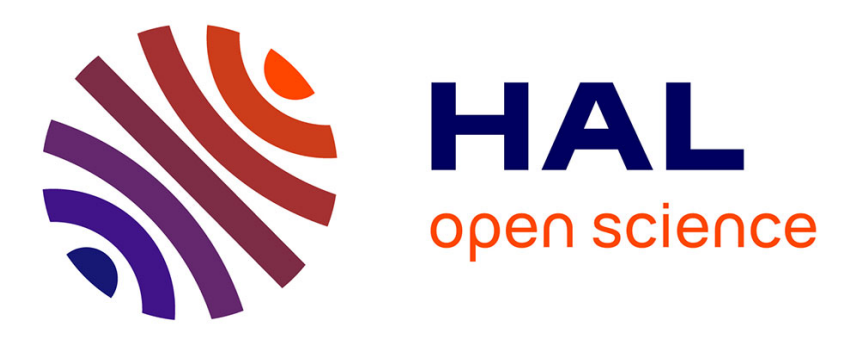

\title{
A AM/FM Single Component Signal Reconstruction using a Nonsequential Time Segmentation and Polynomial Modeling
}

\author{
Meryem Jabloun, Michelle Vieira, Nadine Martin, François Léonard
}

\section{To cite this version:}

Meryem Jabloun, Michelle Vieira, Nadine Martin, François Léonard. A AM/FM Single Component Signal Reconstruction using a Nonsequential Time Segmentation and Polynomial Modeling. International Workshop on Nonlinear Signal and Image Processing, NSIP 2005, May 2005, Sapporo, Japan. hal-00381217

\section{HAL Id: hal-00381217 https://hal.science/hal-00381217}

Submitted on 5 May 2009

HAL is a multi-disciplinary open access archive for the deposit and dissemination of scientific research documents, whether they are published or not. The documents may come from teaching and research institutions in France or abroad, or from public or private research centers.
L'archive ouverte pluridisciplinaire HAL, est destinée au dépôt et à la diffusion de documents scientifiques de niveau recherche, publiés ou non, émanant des établissements d'enseignement et de recherche français ou étrangers, des laboratoires publics ou privés. 


\title{
A AM/FM Single Component Signal Reconstruction using a Nonsequential Time Segmentation and Polynomial Modeling
}

\author{
Meryem JABLOUN*, Michelle VIEIRA*, Nadine MARTIN* and François LEONARD ${ }^{\dagger}$ \\ * LIS-CNRS/INPG, 961 rue de la houille blanche, BP 46, 38402 Saint Martin d'Hères, France. \\ first name.last name@lis.inpg.fr . 'Institut de recherche d'Hydro-Québéc, 1800 Boul. Lionel Boulet, \\ Varennes (QC) Canada, J3X 1S1. leonard.francois@,ireq.ca
}

\begin{abstract}
The problem of estimating nonstationary signals has been considered in many previous publications. In this paper we propose an alternative algorithm in order to accurately estimate $\mathrm{AM} / \mathrm{FM}^{1}$ signals. Only single component signals are considered. We perform local polynomial modeling on short time segments using a nonsequential strategy. The degree of polynomial approximation is limited due to the shortness of each time segment. The time support of a segment is controlled by a criterion defined on the spectrogram. To keep optimality a maximum likelihood procedure estimates the local model parameters leading to a non linear equation system in $R^{7}$. This is solved by a Simulated Annealing technique. Finally, the local polynomial models are merged to reconstruct the entire signal model. The proposed algorithm enables highly nonlinear AM/FM estimation and shows robustness even when Signal to Noise Ratio (SNR) is low. The appropriate Cramer Rao Bounds (CRB) are presented for both polynomial phase and amplitude signals. Monte Carlo simulations show that the proposed algorithm performs well. Finally, our proposed method is illustrated using both numerical simulations and a real signal of whale sound.
\end{abstract}

\section{INTRODUCTION AND OUTLINE}

This paper is concerned with the commonly encountered problem of estimating nonlinear AM/FM signals. This topic is frequently addressed in engineering systems and applied science. Examples of which involve radar, sonar, mechanics, speech, and communications. Although this problem has been widely investigated for many years [2-3-4-9-10-14], the proposed techniques fail to estimate the AM/FM modulation at low SNR. These used AM/FM polynomial models, hence strong modulations need high orders. However, this reduces the performance of the analysis.

So, in aiming, both, to estimate all types of modulation and to improve the accuracy, we present an alternative approach. Contrary to [2-3-4-9-10-14], we propose to pay attention to the local variations of the modulations instead of the entire frequency and amplitude. To keep optimality we locally estimate these variations using a Maximum Likelihood (ML) procedure.

In other words, we segment the signal in short time windows as shown in Fig 1. The first segment corresponds with the most energetic part of the signal; the segmentation is then carried out on both positive and negative time

\footnotetext{
${ }^{1}$ AM/FM: Amplitude Modulation/ Frequency modulation
}

progression. This corresponds to a nonsequential strategy which is an original part of this work. The length of each segment is adapted to locally approximate the amplitude and the frequency by second order polynomial functions in agreement with the Weierstrass theorem. We then estimate parameter models using the ML procedure. As this leads to a rather complicated function to be minimized, we use an optimization technique which is easily implemented and increases efficiency. We will show the effects of the chosen polynomial base upon the parameter estimation. An orthogonal polynomial base results in amplitude parameters being uncoupled. Therefore, we propose one adapted to the discrete time context. In that case we get higher estimation accuracy. Once all local polynomial models have been estimated, we propose a merging strategy to reconstruct the entire signal model.

The suggested method enables us to free ourselves from a polynomial phase and amplitude model as in [2-3-4-9-10-14], for the entire modulation. Therefore, the estimation of the model polynomial order is no longer necessary. We also benefit from the fact that there is no more error propagation due to the iterative estimation of the polynomial coefficients as in [2-3-4-9-10-14]. Moreover the proposed algorithm results in a good compromise between the local optimality and computational time.

The discrete signal to be dealt with herein is given by:

$$
\left\{\begin{array}{l}
y[n]=s[n]+e[n] \\
s[n]=A[n] \mathrm{e}^{\mathrm{j} \Phi[n]}
\end{array} \text { with } 0 \leq n \leq \mathrm{N}-1\right.
$$

Where $e[n]$ is assumed to be a white complex Gaussian process with zero mean and unknown variance $\sigma^{2}$. We assume no discontinuous phase $\phi[\mathrm{n}]$, strictly positive amplitude $A[n]$ and reduced frequency $F[n]$ respecting the Shannon theorem.

Section II describes the local model estimation. In Section III, we develop the time segmentation algorithm. In Section IV, Cramer Rao bounds (CRB) derived in [4] for the entire frequency and amplitude are compared to the proposed global estimator. The analysis of numerical simulations and a real signal are discussed in Section V. Finally Section VI sets out our conclusions and work in progress.

\section{LOCAL MODEL ESTIMATION}


In this section, we consider a short time segment of the component defined in (1). Its time support is obtained from the time-segmentation algorithm introduced later. It is short enough to allow a second order polynomial approximation for both the local frequency and amplitude. Hence, we have a reduced number of coefficients to estimate. Typically, the length ranges between 12 and 60 samples with respect to the variations of the frequency modulation.

\section{A. Local AM/FM Model}

For the $\mathrm{i}^{\text {th }}$ segment, both the local frequency $f_{\mathrm{i}}[k]$ and amplitude $a_{\mathrm{i}}[k]$ (Fig. 2) are approximated by:

$$
\left\{\begin{array}{ll}
a_{i}[k]=\sum_{u=0}^{2} a_{i, u} & g_{i, u}[k] \\
f_{i}[k]=\sum_{u=0}^{2} f_{i, u} & g_{i, u}[k]
\end{array} \text { with }-L_{i} / 2 \leq k \leq L_{i} / 2\right.
$$

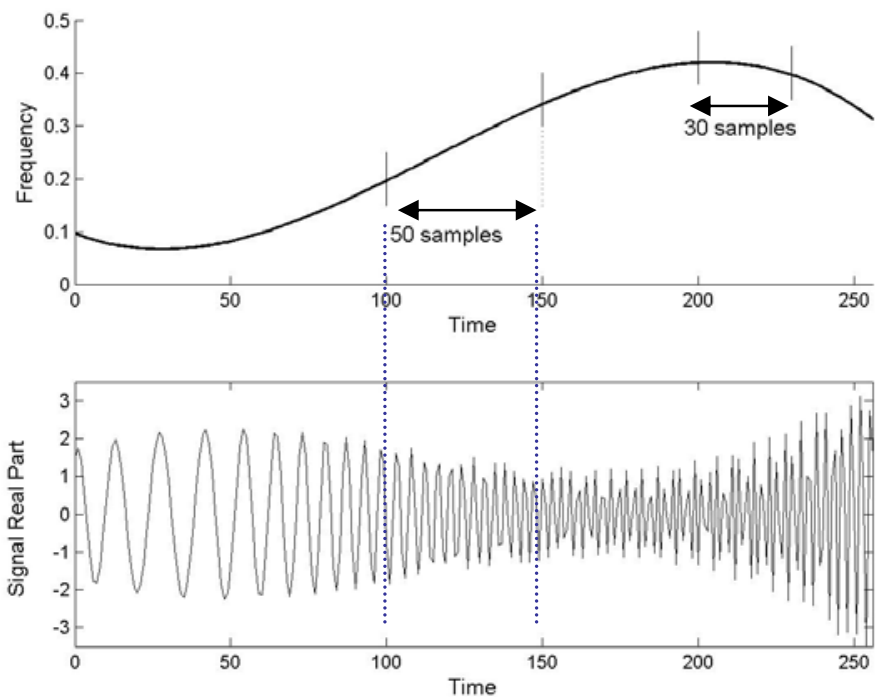

Fig. 1. Examples of segment length for a nonlinear AM/FM signal

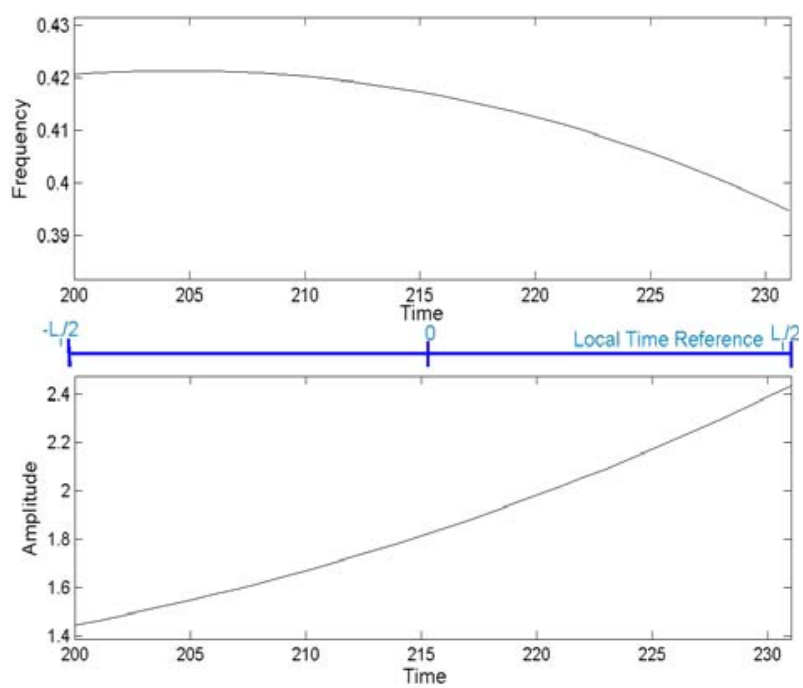

Fig. 2. Local segment of an AM/FM modulation (31 samples). where $g_{i, u}[k]$ is a $\mathrm{u}^{\text {th }}$ order normalized polynomial defined over $\left[-L_{\mathrm{i}} / 2, L_{\mathrm{i}} / 2\right]$. To simplify implementation of the algorithm, the segment length $L_{\mathrm{i}}+1$ is assumed to be odd. Additionally, we reference the local time $k$ and initial phase $\theta_{\mathrm{i}, 0}$ to the segment centre in order to minimize estimation errors [8-11]. Then the phase is locally approximated by:

$$
\phi_{i}[k]=\theta_{i, 0}+2 \pi\left[\sum_{u=L_{i} / 2}^{k} f_{i}[u]-\sum_{u=-L_{i} / 2}^{0} f_{i}[u]\right]
$$

To sum up, for the $\mathrm{i}^{\text {th }}$ segment, we have to estimate $\theta_{i}=\left\{a_{i, 0}, a_{i, 1}, a_{i, 2}, \theta_{i, 0}, f_{i, 0}, f_{i, 1}, f_{i, 2}\right\}$. This is a set of seven parameters: three parameters for the amplitude, the initial phase, and three parameters for the frequency. The estimation is done using two constraints: strictly positive amplitude and no discontinuous phase.

To conserve optimality, a ML technique is used for the local parameter estimation. It results in a nonlinear equation to be minimized in $\mathfrak{R}^{7}$. As the noise is assumed to be Gaussian, the ML estimator is equivalent to the Least Squares (LS) one. So we write:

$$
\hat{\theta}_{i}=\underset{\theta_{i} \in \Re^{7}}{\arg \min } \ell_{L S}\left(\theta_{i}\right)=\sum_{k={ }^{-L_{i}} / 2}^{L_{i} / 2}\left|y_{i}[k]-s_{i}[k]\right|^{2}
$$

Through $y_{\mathrm{i}}[\mathrm{k}]$ and $s_{\mathrm{i}}[\mathrm{k}]$ we can note the noisy observations and the signal model respectively. They are restricted to the $i^{\text {th }}$ segment and time referenced to its centre.

\section{B. Optimization strategies}

Due to the complexity of the likelihood function (4), parameter estimation by direct maximization is extremely difficult. To overcome this problem, we apply two numerical methods: the Simulated Annealing (SA) and the MCMC (Metropolis Hasting random walk one variable at time) method which are stochastic techniques.

The SA one has analogies with the annealing of solids in physics [7]. It has significant efficiency when a desired global extremum is hidden in many local extrema, and its implementation is relatively simple. The MCMC method [1] is also easily implemented. However it requires more time to converge. In [6] we compare both SA and MCMC methods for a quadratic modulation over a short time window. MonteCarlo simulations show the mean square errors (MSE) of the parameter estimates are closed to the derived CRB for both SA and MCMC techniques. Nevertheless, the SA one is less biased than that of the MCMC. We finally choose the SA technique based on these observations in the following.

An important point in reducing the convergence time for the chosen algorithm is to use a good initialization source. So, to solve (4), the time frequency (TF) trajectory provided by the spectrogram is used to initialize the SA technique. This is composed of the ridge of energy. As it is a continuous time function, it has a polynomial approximation. This gives a close initialization for the frequency and amplitude parameters. The remaining parameters are set equal to zero. 


\section{Base effects}

Here the influence of the chosen polynomials is studied. In fact, from results given in [6-4], the Fisher information matrix derived for amplitude parameters is diagonal for orthogonal base only. So, this orthogonality is interesting since it leads to uncoupling of the parameter estimation. Thus the estimation accuracy will be improved. An orthogonal polynomial base is given by Legendre one in continuous time. However when discretized it looses its orthogonal properties. So, we derive a discrete orthonormal polynomial base in discrete time directly.

Let $\mathbf{g}_{\mathrm{i}, \mathrm{u}}=\left[g_{\mathrm{i}, \mathrm{u}}\left[-L_{\mathrm{i}} / 2\right], \ldots, g_{\mathrm{i}, \mathrm{u}}\left[L_{\mathrm{i}} / 2\right]\right]$ be a $L_{\mathrm{i}}+1$ dimension orthonormal vector such as:

where $\left\{\begin{array}{l}\quad\left\langle\mathbf{g}_{\mathrm{i}, \mathrm{u}}, \mathbf{g}_{\mathrm{i}, \mathrm{v}}>=\delta_{\mathrm{u}, \mathrm{v}}\right. \\ g_{i, 0}[k]=\kappa_{0}^{0}[k]=\kappa_{1}^{0}+\kappa_{1}^{1} k \quad \text { with } k \in\left[-L_{i} / 2, L_{i} / 2\right] \\ g_{i, 1}[k]=\kappa_{2}^{0}+\kappa_{2}^{1} k+\kappa_{2}^{2} k^{2}\end{array}\right.$

$\kappa_{u}^{v}$ is the $\mathrm{v}^{\text {th }}$ order polynomial coefficient of $g_{\mathrm{i}, \mathrm{u}}[\mathrm{k}]$. By applying the Gram-Schmidt procedure over $\left[-L_{\mathrm{i}} / 2, L_{\mathrm{i}} / 2\right]$ using (5), we get the $\kappa_{u}^{v}$ values

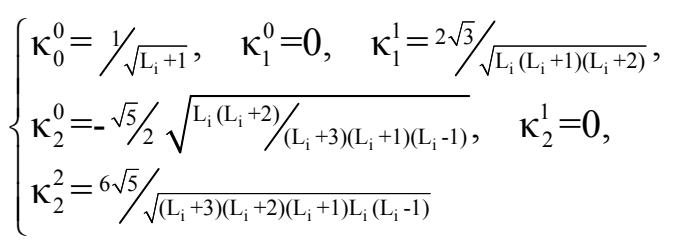

Then three different bases are compared: the canonical base $^{2}$ which is not normalized, the discretized Legendre base and the proposed one (7). Fig. 3. shows the Mean Square Error (MSE) of $a_{0, \mathrm{i}}$ and $f_{0, \mathrm{i}}$ estimates using the three bases. Fig. 4. shows their bias. The same quadratic AM/FM modulation is considered over a 33-sample segment. Both of Fig. 3 and 4 illustrate the higher estimation given by the proposed base. This remark is true for the remaining parameters too. This helps the SA technique in finding the shortest path to the global extremum, hence increasing the convergence rate of the algorithm. Moreover orthogonality simplifies the initialization of the agitation low [13-7] of the SA algorithm.

Considering the time-support shortness, the SA algorithm for the estimation of the parameters is biased (Fig4). As the $\mathrm{CRB}$ is the lower bound on the estimation accuracy of an unbiased estimator, estimation errors are not directly comparable with the CRBs given in [4-6]. Here, it is just used as a reference in evaluating the performance of the algorithms.

\section{Time Segmentation Algorithm}

To find the time support of each segment we proceed in three steps:

First, the main energy peak of the spectrogram is located in time and frequency. This corresponds with the most energetic

\footnotetext{
2 The second order canonical base: $1, \mathrm{x}$ and $\mathrm{x}^{2}$, for $\mathrm{x}$ belongs to $\left[-\mathrm{L}_{\mathrm{i}} / 2, \mathrm{~L}_{\mathrm{i}} / 2\right]$
}

point of the signal. The spectrogram is used to do this since the frequency resolution is not critical. The segment length is
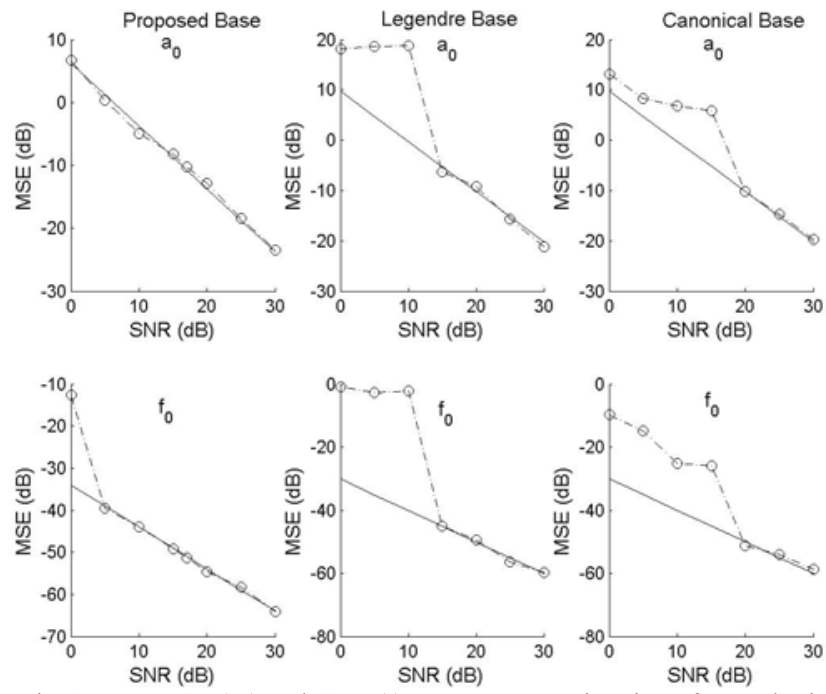

Fig. 3. MSE (o-) and CRB (-): Parameter estimation of a quadratic AM/FM signals using different bases: the orthonormal proposed base, the Legendre base and the canonical one from left to right respectively.
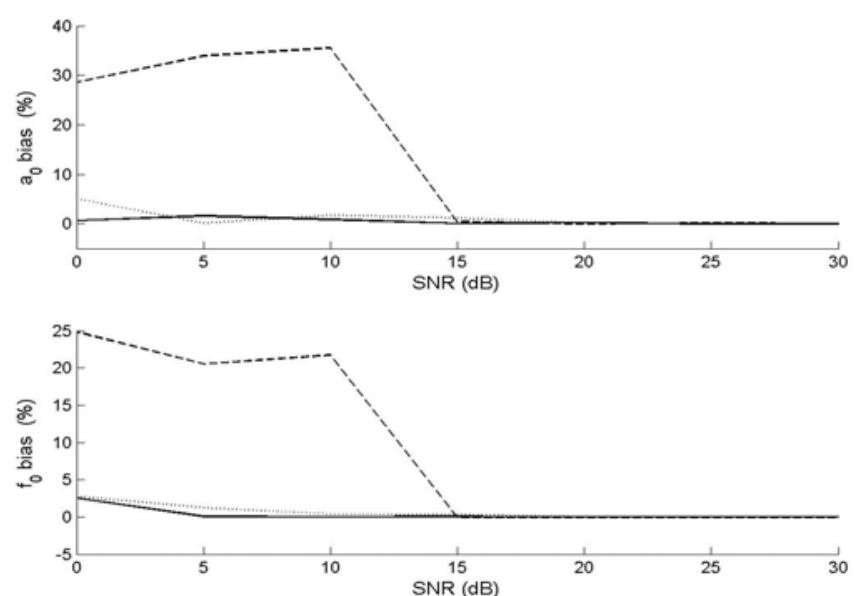

Fig. 4. Bias in (\%) of the parameter estimates of a quadratic AM/FM signals using different bases: the orthonormal calculated base (-), the Legendre base (:) and the Canonical one (- -)

fixed to at least three periods of the main peak frequency. We also limit the sample number to 60 to reduce the time convergence.

Then local estimation of the frequency and the amplitude is done using the method given in Section II, see Ref. [13] for the implementation of the SA-method. Following this all other segments are extracted and treated in the same way by locating next energetic peaks.

Finally we reconstruct the entire signal by merging the contiguous local models. We need to detect the overlap between segments. In doing so, the frequency is smoothed by a Hamming window with respect to the phase continuity constraint. Fig. 5. shows the significant weight of the Hamming windows in the segment centre since the error estimation is minimized there. 


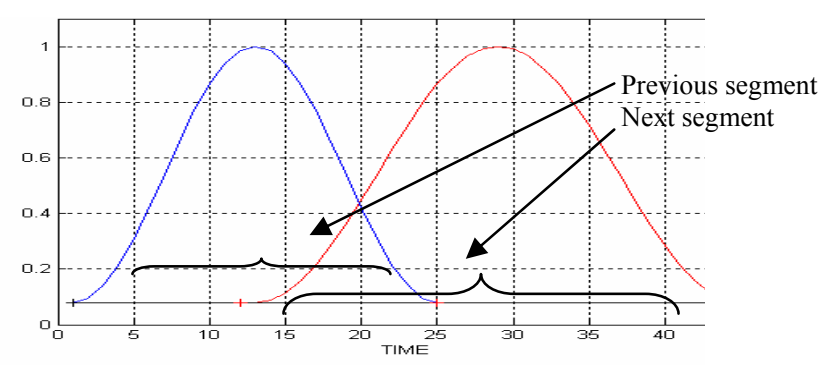

Fig. 5 : Smoothing using hamming windows

The steps involved in the entire signal estimation are as follows:

Initialization Let $i=0, \hat{y}[n]=y[n]$, for $0 \leq n \leq N-1$

Step 1 Determine the segment to be processed.

a) $\left[n_{\max }, f_{\max }\right]=\underset{n, f}{\arg \max }|\operatorname{spectrogram}(\hat{y}[n])|$

b) Set $\mathrm{L}_{\mathrm{i}}=3 *$ floor value of $\left(1 / f_{\max }\right)$.

c) Put segment $\mathrm{i}_{\mathrm{i}}=\left[\mathrm{n}_{\max }-\mathrm{L}_{\mathrm{i}} / 2, \mathrm{n}_{\max }+\mathrm{L}_{\mathrm{i}} / 2\right]$

Step 2 Estimate the local polynomial model as in section II.

Step 3 If there is overlap between the estimated segments smooth for both the local frequency and amplitude ( Fig. 5.). Otherwise go to step4.

Step 4 If all samples are treated go to step 5, else set $i=i+1$. Reconstruct the estimated parts of the signal model using (1).

Remove them from $\hat{y}[\mathrm{n}]$. Then restart step 1 .

Step 5 Reconstruction of the entire estimate signal model using (1).

We notice that the model given by (1) is still conserved since the estimated parts of the signal model are not removed when we use (4). This avoids error accumulation due to the segmentation.

The proposed strategy is nonsequential. It is particularly interesting in that it constructs segments centered on the most energetic points of the signal. This considerably decreases estimation errors. Furthermore, the proposed algorithm is well balanced with respect to accuracy and low CPU time. In spite of its apparent complexity, it is easy to implement. For example for a cubic AM/FM modulation with $\mathrm{N}$ equal to 257 samples, we have about five segments to process depending on the SNR

Contrary to the existing techniques such as the HAF (Higher Ambiguity Function) [9-4-3-2], we don't need to estimate degree and the coefficients of the entire AM/FM models. Local variations of the frequency and the amplitude are closely tracked. Examples in Section V show good results. Moreover, Ref. [6] noted that in a comparison with HAF for polynomial phase signals, the proposed algorithm shows the best tracking of the AM/FM modulations.

\section{CRAMER RAO BOUND}

Let us consider a polynomial phase and amplitude signal with a single component. The signal model is given by (1). The amplitude and the frequency are given by the following equations:

$$
\left\{\begin{array}{l}
A[n]=\sum_{u=0}^{P} a_{u} \rho_{u}[n] \\
\Phi[n]=\sum_{u=0}^{Q} b_{u} \eta_{u}[n]
\end{array} \text { for } 0 \leq n \leq N-1\right.
$$

where $\rho_{\mathrm{u}}[\mathrm{n}]$ and $\eta_{\mathrm{u}}[\mathrm{n}]$ are normalized canonic polynomial bases. $\mathrm{P}$ and $\mathrm{Q}$ are polynomial orders of the amplitude and the phase respectively. In [4], Friedlander and Francos derived the CRB for polynomial amplitude and phase for the entire modulation.

$$
\left\{\begin{array}{l}
C R B(A[n])=\frac{\sigma^{2}}{2} \boldsymbol{\rho}^{H}\left\{\boldsymbol{\Phi}^{H} \boldsymbol{\Phi}\right\}^{-1} \boldsymbol{\rho} \\
C R B(F[n])=\frac{\sigma^{2}}{2} \mathbf{h}^{H}\left\{\boldsymbol{\Lambda}^{H} \boldsymbol{\Lambda}\right\}^{-1} \mathbf{h}
\end{array}\right.
$$

with $\rho=\left[\rho_{0}[n], \ldots, \rho_{\mathrm{P}}[\mathrm{n}]\right], \quad \Lambda=\mathrm{j}\left[\eta_{0}(\mathbf{n}) . \mathbf{s}(\mathrm{n}), \ldots, \eta_{\mathrm{Q}}(\mathbf{n}) . \mathbf{s}(\mathbf{n})\right]$, $\mathbf{h}=1 / 2 \pi\left[\eta_{0}^{\prime}[\mathrm{n}], \cdots, \eta_{\mathrm{Q}}^{\prime}[\mathrm{n}]\right]$,

and $\quad \boldsymbol{\Phi}=\left[\rho_{0}(\mathbf{n}) \cdot \mathrm{e}^{\mathrm{j} \Phi(\mathrm{n})}, \ldots, \rho_{\mathrm{P}}(\mathbf{n}) \cdot \mathrm{e}^{\mathrm{j} \Phi(\mathrm{n})}\right]$

The symbol of ' $\bullet(\mathbf{n})$ 'denotes the $\mathrm{N}$ dimension vector of a scalar function ' $\bullet$ (n)' for $0 \leq n \leq N-1$. ' .'denotes element by element multiplication of the vector entries. ${ }^{\mathrm{H}} \mathrm{H}$, is the transpose conjugate and ' ' , is for the derivation.

We observe that the derived CRB are functions of time. To make comparison with the CRBs given by (9), we choose a cubic polynomial AM/FM signal, as an example, with $\mathrm{N}=257$, see Fig. 6. Monte Carlo simulations with 100 trials were run with global ${ }^{3} \mathrm{SNR}$ equal to $15 \mathrm{~dB}$ and $10 \mathrm{~dB}$. Fig 7 and 8 illustrate the MSE of the reconstructed amplitude and frequency modulations versus the corresponding CRBs (9) respectively. They show a robustness of our proposed technique when the SNR becomes low.

It is important to note that the SNR is not constant over time due to the nonstationarity of the signal. Therefore, the SNR can be locally ${ }^{4}$ lower than the global SNR which explains the differences in performance over time (Fig. 9).

\section{NUMERICAL EXAMPLES}

In this section, the proposed algorithm is illustrated with numerical examples.

First we use a fifth order polynomial AM/FM signal which means that the phase order is 6 . This is a very high AM/FM modulation. Fig. 10 shows three curves: the original signal versus the reconstructed ones with SNR equal to 15 and 10 $\mathrm{dB}$ and for $\mathrm{N}=257$. It is easily seen that the estimated curves are close to the original amplitude and frequency. Thus the proposed algorithm is able to estimate highly modulation.

\footnotetext{
3 A global SNR is evaluated using the entire signal

${ }^{4}$ A local SNR is evaluated on each segment
} 


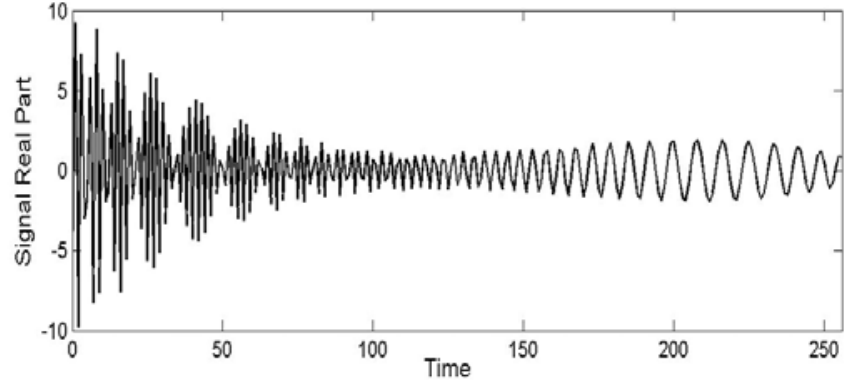

Fig. 6: An cubic $-\mathrm{AM} / \mathrm{FM}$ signal with $\mathrm{N}=257$ and parameter values $\left\{\mathrm{a}_{0}, \mathrm{a}_{1}, \mathrm{a}_{2}, \mathrm{a}_{3}, \theta_{0}, \mathrm{~b}_{0}, \mathrm{~b}_{1}, \mathrm{~b}_{2}, \mathrm{~b}_{3}\right\}=\{18.7,4.7,32.0,-31.6 ;-2.18,4.8,-3.5,0.15,1.57\}$.
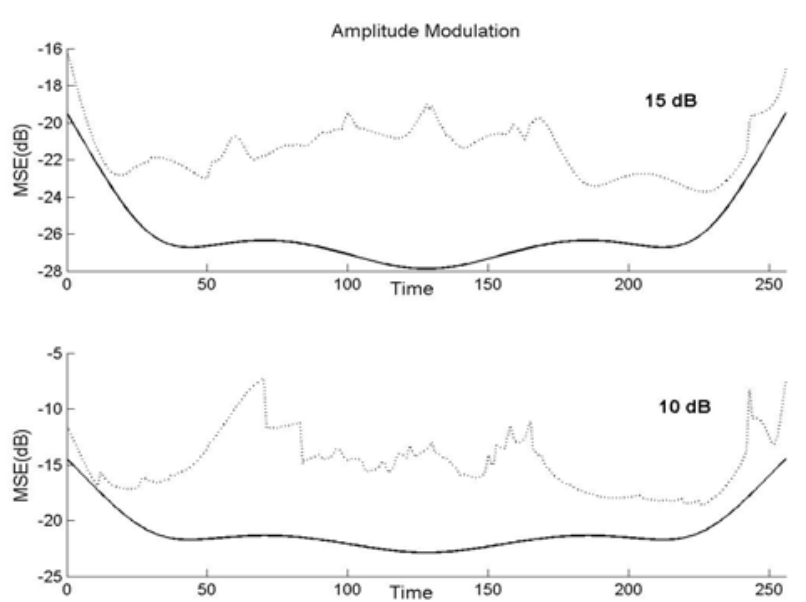

Fig. 7 : CRB (-) versus amplitude MSE (:) for the cubic AM/FM signal of the Fig. 6. for $\mathrm{SNR}=15 \mathrm{~dB}$ and $10 \mathrm{~dB}$.
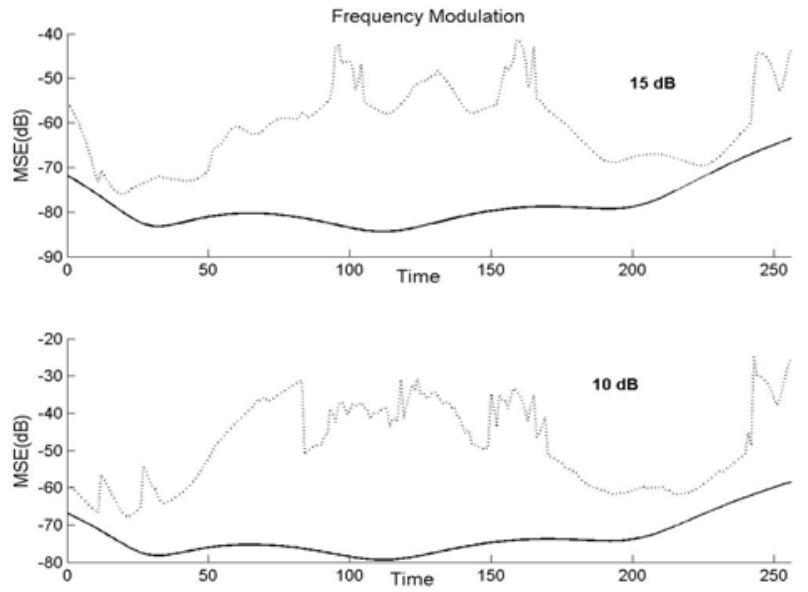

Fig. 8 : CRB (-) versus frequency MSE (:) for the cubic AM/FM signal of the Fig. 6. for $\mathrm{SNR}=15 \mathrm{~dB}$ and $10 \mathrm{~dB}$.



Fig. 9: Local SNR (dB) computed on 25 samples gliding window for a global $\mathrm{SNR}=15 \mathrm{~dB}$



(b) Amplitude Modulation

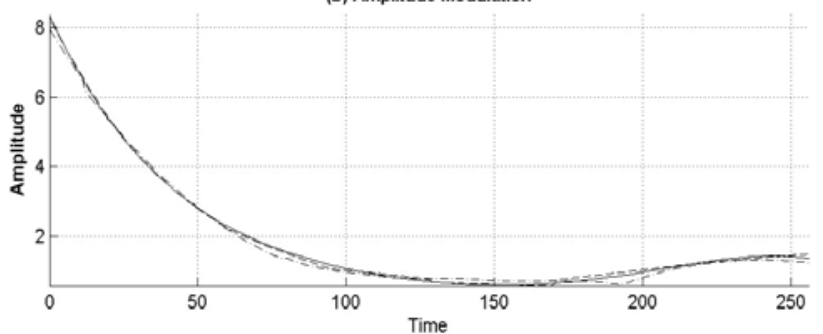

Fig. 10 : (a) Original frequency (-) versus the reconstructed ones for $15 \mathrm{~dB}$ (-- ) and for $10 \mathrm{~dB}($ : ). (b) Original amplitude versus the reconstructed ones for $15 \mathrm{~dB}$ and $10 \mathrm{~dB}$

Then we consider an other example: a sinusoidal AM/FM signal as in (1) with

$$
\left\{\begin{array}{l}
A[n]=5+5 \sin (\pi n / N) \\
F[n]=0.25+0.1 \cos (2 \pi n / N)
\end{array} \text { with } 0 \leq n \leq N-1\right.
$$

We note that it is not polynomial phase. Fig. 11 shows it to be well estimated for SNR equal to 15,10 and $5 \mathrm{~dB}$. This illustrates that we can apply local polynomial models to estimate a nonpolynomial phase signals.

Finally to show the efficiency of the algorithm we consider a real signal: that of whale sound. In certain bioacoustics studies on the effects of human-made sound on a whales' behavior, scientists need estimate the whale sounds embedded in significant noise, particularly that due to shipping. Far from simple linear chirps and being affected by amplitude distortion, detection of the whale's sound need more than


Fig. 11 : (a) Original frequency (-) versus the reconstructed ones: (--) for $15 \mathrm{~dB},(:)$ for $10 \mathrm{~dB}$, and (-.-) for $5 \mathrm{~dB}$. (b) Original amplitude versus the reconstructed ones: for $15 \mathrm{~dB}$, for $10 \mathrm{~dB}$ and for $5 \mathrm{~dB}$. 
linear AM/FM-estimation techniques. Fig. 12 shows the spectrogram of the noisy signal. As we see the information gives by the spectrogram or Fourier transform does not yield precise information for the AM and FM modulation. Fig. 13 and 14 give the result of our approach for unknown SNR. Most of the frequency variations seem to be in agreement with the spectrogram. The estimated amplitude thought of as the mean of the noisy observations.

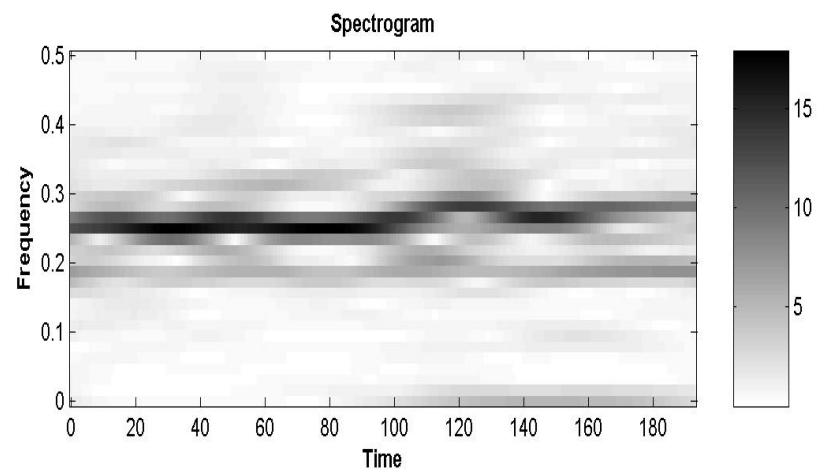

Fig. 12: Spectrogram of the whale sound



Fig. 13 Estimated Frequency modulation

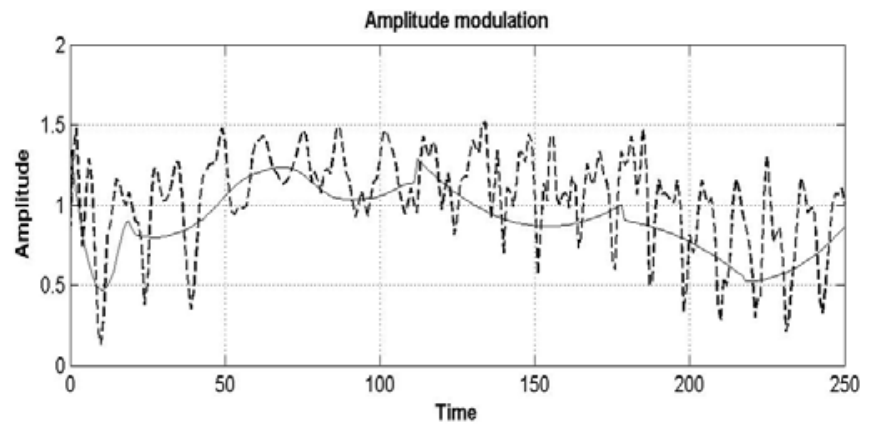

Fig. 14 Estimated-Amplitude modulation (-) versus the amplitude of Hilbert transform of the original noisy signal (- -).

\section{CONCLUSIONS}

In this paper we investigate the estimation of highly nonstationary single component signal embedded in noise. Based on nonsequential time segmentation and local polynomial modeling, the proposed algorithm is able to fit the frequency and the amplitude by merging local polynomial models. Using a maximum likelihood estimator for the local model parameters allow us to keep optimality. Comparisons with existing techniques such HAF show that our approach is performant [6]. It is not limited to linear AMFM modulation. Moreover, it doesn't need prior estimation on the phase degree. Numerical simulations, including sinusoidal AM/FM modulation and high order polynomial phase signals, show the good performance. Aiming now to study multicomponent signals works are in progress in this direction.

\section{ACKNOWLEDGMENT}

We want to thanks Dr C. Ioana, ENSIETA, FRANCE, to help finding real signals.

\section{REFERENCES}

[1] S. Chib and E. Greenberg. "Understanding the Metropolis Hastings algorithm. The American statician," vol 49, 1995, pp 327-335.

[2] S. Barbarossa, A. Scaglione and G. B. Giannakis , " Product higher order function for multicomponent polynomial phase signal modeling ", IEEE Trans. On Signal Processing vol. 49, NO. 3, March 1998, pp. 691-708.

[3] A.Francos and M. Porat, "Analysis and Synthesis of Multicomponent Signals using Psitive Time-Frequency Distributions," ", IEEE Trans. on Signal Processing, vol. 47, no. 2, pp 493-504, April 1995.

[4] B. Friedlander and J.M. Francos, "Estimation of Amplitude and Phase parameters of Multicomponent Signals", IEEE Trans. on Signal Processing, vol. 43, no. 4, pp 917-926, April 1995.

[5] S. Golden and B. Friedlander , " Maximum likelihood estimation, Analysis and applications of exponential polynomials signals", IEEE Trans. On Signal Processing vol. 47, NO. 6 pp. 1493-1501, june 1999.

[6] M. Jabloun, M. Vieira, N. Martin and F. Leonard. "Local Orthonormal Decomposition for both Instantenous Amplitude and Frequency of highly nonstationary discrete Signals," IMA: International conference for Mathematics and its Applications Cirencester December 2004, in press.

[7] P. J. M. Laarhoven and E. H. L. Aarts "Simulated Annealing: Theory and Applications," Kluwer Academic Publishers, 1987.

[8] F. Leonard. "Referencing the phase to the center of the spectral window why?," Mechanical System and Signal Processing, 1997, vol. 11, No. 1,pp 75-90.

[9] S. Peleg and B. Friedlander, "The Discrete Polynomial-Phase Transform," IEEE Trans. on signal processing, vol 43, No. 8, August 1995, pp 1901-1914.

[10] S. Peleg, B. Porat and B. Friedlander, "The achievable accuracy in estimating the instantaneous phase and frequency of a constant amplitude', IEEE Trans. On Signal Processing, vol.71, no. 6, pp 2216-2224, June 1993.

[11] C Rife., R. Boorstyn, "Single-Tone Parameter Estimation from Discrete-time Observation", IEEE Transactions on information theory, vol. IT-20, no. 5,1974.

[12] S. Saha and S. M. Kay, "Maximum likelihood parameter estimation of superimposed chirps using Monte Carlo importance sampling", IEEE Trans. On Signal Processing, vol. 50, no.2, pp 224-230, February 2002.

[13] M. Vieira, F. Leonard, M. Jabloun and N. Martin., " Short time single polynomial phase using Legendre functions", EUSIPCO, September 6-10, 2004, pp793-796.

[14] G. Zhou, G.B. Giannakis and A. Swami, "On polynomial phase signals with time-varying amplitudes", IEEE Transactions on Signal Processing, vol. 44, no. 4, April 1996, pp 848-861 